\title{
More power to their elbow
}

\author{
Fiona Godlee editor in chief
}

The BMJ

Bones and joints seem to be featuring more than usual in The $B M J$ 's research and education sections. This is a good thing. They represent a large and growing part of the work of healthcare, as people live longer and, hopefully, more active lives. And the presence of these studies in The BMJ indicates that the field is on the up, with stronger research methodology and a greater willingness to answer questions and report on outcomes that matter to patients.

A couple of weeks ago we heard that subachromial decompression surgery offered no important improvements in pain, function, or quality of life when compared with non-operative management and carried greater risk of serious harm (doi:10.1136/bmj.1294)

And Anthony Palmer and colleagues reported their multicentre pragmatic randomised trial comparing arthroscopic hip surgery with physiotherapy in people with femoroacetabular impingement (doi:10.1136/bmj.1185). The authors used validated patient reported outcomes and blinded clinical assessment, but this was a relatively small trial (222 patients) with a shortish follow-up period of six months after surgery. Their conclusion-that surgery gives better results-must await confirmation from a systematic review combining this with two other recent trials.

This week we publish two further studies on orthopaedic procedures. First come Hannah Wilson and colleagues with their systematic review and meta-analysis comparing unicompartmental and total knee replacement for people with end stage knee osteoarthritis (doi:10.1136/bmj.1352). By combining limited data from randomised trials with evidence from national joint registries, databases, and cohort studies, they conclude that both options are viable. Unicompartmental arthroplasty offers shorter hospital stays, fewer medical complications, and better functional outcomes than knee replacement but a higher risk of requiring revision surgery within five years.

Second, a look at adverse effects, including the need for revision surgery, in patients undergoing elective shoulder replacement. Acknowledging that randomised controlled trials are hard to do in orthopaedic surgery, Richard Craig and colleagues have analysed routine NHS data on more than 58000 patients (doi:10. $1136 /$ bmj.1298). They find that early serious adverse effects are more common than previously realised, occurring in nearly 5\% of patients. The risks increased with age and comorbidity, and were higher in men. A patient's lifetime risk of needing revision surgery was highest in younger men (aged under 60) and lowest in older women (over age 85). Most revisions happened within five years of surgery.

In a comment on their study on BMJ Opinion the authors describe "a growing momentum, enthusiasm, and commitment within surgical communities-particularly in orthopaedic surgery, to address remaining evidence gaps" (https://blogs.bmj. $\mathrm{com} / \mathrm{bmj} / 2019 / 02 / 20 /$ providing-better-evidence-orthopaedicsurgery-rcts-registries-both). We have always known that The $B M J$ isn't about teaching orthopaedic surgeons how to do orthopaedic surgery. Our job is to help them and other specialists maintain a broad awareness of advances in medicine as a whole. But understanding the benefits and risks of orthopaedic procedures has become everyone's business. The more high quality research in this area the better. 